\title{
Clinical Signs, Computed Tomographic Imaging, and Management of Chronic Otitis Media/Interna in Dairy Calves
}

\author{
Jérôme Van Biervliet, Gillian A. Perkins, Brett Woodie, Alessandra Pelligrini-Massini, Thomas J. Divers, and
} Alexander de Lahunta

$\mathbf{A}^{3}$ 31/2-week-old Holstein heifer calf was presented to A Cornell University Hospital for Animals (CUHA) with a 2-week history of intermittent fever, coughing, and decreased growth and a 1-week history of drooling and droopy ears. The calf had been treated with several antibiotics without marked improvement. On physical examination, the calf was depressed, underweight, bradycardic (heart rate $60 \mathrm{bpm}$ ) and febrile $\left(103.4^{\circ} \mathrm{F}, 39.7^{\circ} \mathrm{C}\right)$. Clinical signs attributable to cranioventral pneumonia included bilateral mucopurulent nasal discharge, coughing, and abnormal bronchial tones. Intermittently, there were food particles in the nasal discharge, ptyalism, difficult prehension and mastication of food, and regurgitation of green material from both the nose and mouth. Neurologic examination revealed depression, bilateral facial nerve paresis (decreased lip, eyelid, and ear tone; absent palpebral or menace reflex), and bilateral vestibular disease (balance loss to either side, no head tilt, and loss of physiologic vestibular nystagmus). No abnormal nystagmus was observed, but the eyeballs did not show the physiologic vestibular eyedrop on head and neck extension. The gait was normal. The diagnosis of bilateral cranial nerve (CN) VII and VIII disease suggested bilateral otitis media/interna. Deep palpation of the base of the ears elicited a pain response and nonodorous otorrhea was present, indicating a component of otitis externa. Upper airway and esophageal endoscopy was consistent with dysphagia and disturbed esophageal motility and showed tracheal mucopus, nasopharyngeal collapse, dorsal displacement of the soft palate, and esophageal dilatation with few contractions. Thoracic radiography and transtracheal aspirate cytology (many degenerate neutrophils, few macrophages, large amounts of mucus, no bacteria) confirmed the presence of bronchopneumonia. The transtracheal aspirate yielded Arcanobacter pyogenes and Mycoplasma spp., but fluorescent antibody testing for common respiratory viruses was negative. CBC results were normal. Lumbosacral cerebrospinal fluid (CSF) had a slightly high nucleated cell count $(9$ nucleated cells $/ \mu \mathrm{L}$, normal $<5$ cells/ $\mu \mathrm{L}$; total protein concentration $17 \mathrm{mg} / \mathrm{dL}$, normal $<70 \mathrm{mg} / \mathrm{dL}$ ). Lateral skull radiographs did not show abnormalities, but computed tomography (CT) imaging indicated that both tympanic bullae and the right petrous temporal bone were enlarged,

From the Department of Clinical Sciences, College of Veterinary Medicine, Cornell University, Ithaca, NY.

Reprint requests: Dr Jerome L.N. Van Biervliet, Centrum voor Menselijke erfelijkheid, Onderzoek, en Navorsing, Faculteit Geneeskunde, Katholieke Universiteit Leuven, Herestraat 49 bus 602, B-3000 Leuven, Belgium; email: jerome.vanbiervliet@med.kuleuven.ac.be.

Received November 10, 2003; Revised January 20, 2004; Accepted April 9, 2004.

Copyright (C) 2004 by the American College of Veterinary Internal Medicine

0891-6640/04/1806-0018/\$3.00/0 thickened, and multifocally lytic, indicating osteomyelitis and otitis interna. Additionally, in both tympanic cavities and external ear canals there was an increased soft tissue density, indicative of bilateral otitis media (Fig 1). An atlanto-occipital CSF fluid sample provided evidence of meningitis (36 nucleated cells/ $\mu \mathrm{L}$, mostly macrophages with moderate lymphocytes and moderate neutrophils, no bacteria, total protein concentration $31 \mathrm{mg} / \mathrm{dL}$ ). The calf was treated with fluids intravenously, nonsteroidal anti-inflammatory drugs, topical nonantibiotic eye ointment, vitamin E and selenium (204 IU vitamin E, $3 \mathrm{mg}$ IM once sodium selenite), and oxytetracycline $(6.6 \mathrm{mg} / \mathrm{kg}$ IV q12h for a total for 14 days). Otitis externa was treated with instillation of diluted vinegar solution in both ears $(50 \%$ distilled vinegar: $50 \%$ water q12h). The calf was able to pan-feed from the ground safely and was given small volumes of milk replacer multiple times per day. Slow but gradual improvement was noticed in all her clinical signs. Endoscopy after 12 days was normal. The calf was continued on oxytetracycline $(20 \mathrm{mg} / \mathrm{kg}$ IM q48h) and procaine-penicillin (22,000 IU/kg IM q12h) for an additional 10 days after discharge. All clinical signs resolved completely and she became a productive animal in the herd.

A 6-week-old Holstein heifer calf was presented to the CUHA with a several-week history of intermittent fever, coughing, nasal discharge, weight loss, depression, and a droopy left ear. The calf had been treated with multiple antibiotics and continued to be febrile. Physical examination revealed a thin body condition, lethargy bordering on obtundation, and fever $\left(102.8^{\circ} \mathrm{F}, 39.3^{\circ} \mathrm{C}\right)$. No clinical signs of pneumonia were found on thoracic auscultation and ultrasonography. Neurologic examination revealed a left head tilt and paralysis of the left lip, eyelid, and ear muscles. When menaced in the left eye, she retracted the eyeball but had no palpebral fissure closure. Her gait was normal but evidenced intermittent loss of balance to the left side, especially after sudden direction change or blindfolding. A neuroanatomic diagnosis of a left peripheral CN VII and VIII deficit was made, consistent with otitis media/interna. In addition to accumulating chewed food on the left side of the mouth, she showed intermittent ruminal regurgitation, along with difficult prehension and mastication. Deep palpation of the base of the left ear elicited a pain response and mucopurulent otorrhea was present on the left side. $\mathrm{CBC}$ results were normal. Because of financial limitations, no further diagnostic tests were performed and treatment with oxytetracycline $(6.6 \mathrm{mg} / \mathrm{kg}$ IV q12h) was initiated, along with fluids intravenously, vitamin E/selenium (204 IU vitamin E, $3 \mathrm{mg}$ IM once of sodium selenite), nonsteroidal anti-inflammatory drugs, and nutritional support (small volumes of milk replacer pan-fed from the ground). Over the following 9 days, the calf's clinical signs improved dramatically. She was afebrile and the ruminal regurgitation 

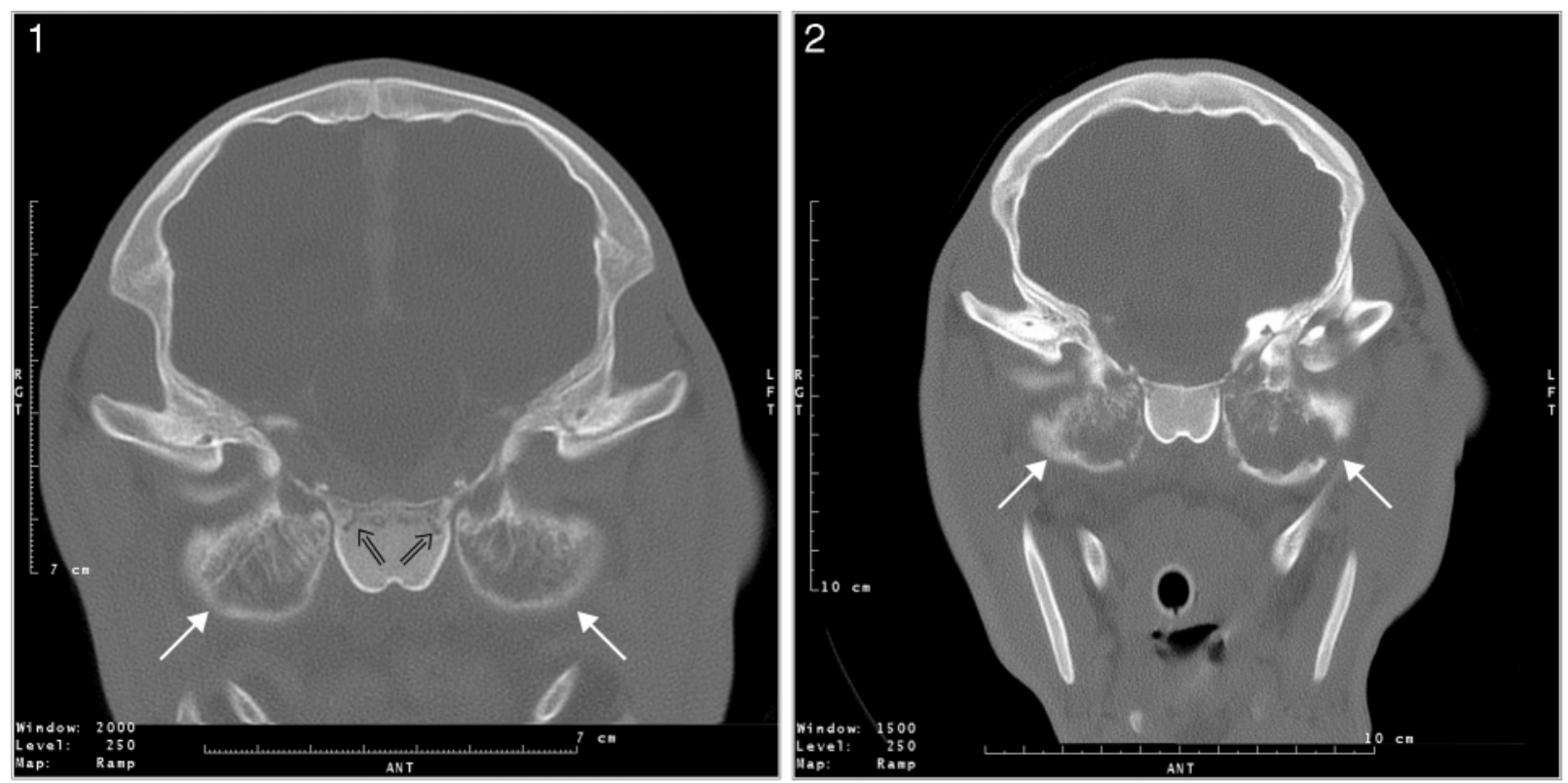

Fig 1. Calf 1. Computed tomographic scan image, bone window. Both tympanic bullae are enlarged, filled with increased soft tissue density (likely fluid), and thickened with multiple areas of moderate to extensive lysis (arrows). Additionally, the basiocciptal bone shows areas of lysis, possibly indicative of osteomyelitis (open arrows).

Fig 2. Calf 2. Computed tomographic scan image, bone window. Both tympanic bullae are enlarged, filled with increased soft tissue density (likely fluid), and thickened with multiple areas of moderate to extensive lysis (arrows).

stopped. Treatment was continued on the farm for another 10 days. The calf was clinically normal at 1 year of age.

A 9-day-old Holstein heifer calf was presented to the CUHA for diarrhea and depression of 4 days duration. During physical examination, she was found to be febrile $\left(103.6^{\circ} \mathrm{F}, 39.8^{\circ} \mathrm{C}\right)$, tachycardic (heart rate $\left.104 \mathrm{bpm}\right), 8-10 \%$ dehydrated, and depressed, and she was passing profuse mucoid yellow diarrhea and had an inducible cough. At this time, no $\mathrm{CN}$ deficits were noticed and no clinical signs of pneumonia were present on the basis of thoracic auscultation and ultrasonography. $\mathrm{CBC}$ and serum biochemistry analyses yielded results consistent with diarrhea (dehydration, neutrophilic leukocytosis, neutrophilia, hyponatremia, hypochloremia, hyperkalemia, low-grade metabolic acidosis), and multiple etiologies were identified (positive fecal antigen capture enzyme-linked immunosorbent assay test for bovine coronavirus, positive latex agglutination test for bovine rotavirus, and positive cultures for E coli, Clostridium perfringens). Whole-blood polymerase chain reaction testing for bovine viral diarrhea, fecal flotation for Cryptosporidium, and fecal culture for Salmonella infections were negative. The calf was treated supportively with fluid therapy, nonsteroidal anti-inflammatory drugs, vitamin E and selenium (204 IU vitamin E, $3 \mathrm{mg}$ IM once of sodium selenite), antibiotics (ceftiofur $2.2 \mathrm{mg} / \mathrm{kg}$ IV q12h and procaine penicillin 22,000 IU/kg IM q12h), and nutritional support (milk replacer at $8 \%$ of body weight via bottle in 4 feedings). Clinical signs of cranioventral bronchopneumonia developed, and a transtracheal aspirate showed evidence of a suppurative bronchopneumonia. Culture of this sample yielded only Mycoplasma spp., and the antibiotic therapy was changed to oxytetracycline $(6.6 \mathrm{mg} / \mathrm{kg}$ IV q12h). Starting on the 3rd day, the calf developed clinical signs compatible with bilateral peripheral CN VII and VIII deficits (bilateral ear, lip, and eyelid paresis; bilaterally absent menace and palpebral reflexes; normal gait; balance loss to either side) suggestive of bilateral otitis media/ interna. The calf became more depressed and had dysphagia, spontaneous regurgitation of milk, and difficulty in nursing from a bottle or prehending food. Lumbosacral CSF sample cytology was normal on day 2 (2 nucleated cells/ $\mu \mathrm{L}$, total protein $17 \mathrm{mg} / \mathrm{dL})$. Endoscopy indicated collapse of the nasopharynx, dorsal displacement of the soft palate, and a widely dilated, hypomotile esophagus. Radiographs of the tympanic bullae were inconclusive. Severe lysis of both tympanic bullae with soft tissue densities in both tympanic cavities and external ear canals were confirmed by CT imaging (Fig 2). The brain and meninges appeared normal. An atlanto-occipital CSF sample (day 5) showed evidence of meningitis (93 nucleated cells/ $\mu \mathrm{L}$, many macrophages, few lymphocytes, few neutrophils, no bacteria, total protein concentration $24 \mathrm{mg} / \mathrm{dL}$ ). External ear swabs from both sides failed to yield any organisms on aerobic, anaerobic, and Hayflick's media. Because of persistent fevers, antibiotic therapy was changed to florfenicol $(20 \mathrm{mg} / \mathrm{kg} \mathrm{IM}$ $\mathrm{q} 48 \mathrm{~h})$ for 48 hours, then to erythromycin $(6.6 \mathrm{mg} / \mathrm{kg} \mathrm{IV}$ $\mathrm{q} 8 \mathrm{~h}$ as a 2-hour infusion) and trimethoprim-sulfamethoxazole $(20 \mathrm{mg} / \mathrm{kg}$ PO q12h mixed in milk replacer) with aural treatment (Nystatin, neomycin sulfate, neomycin base, thiostrepton, triamcinolone acetonide ointment) without improvement. Because she became inappetant, she was started on partial parenteral nutrition (recipe for 24 hours: 1,500 $\mathrm{ml} 8.5 \%$ amino acids with electrolytes [Travasol ${ }^{\circledR}$ ], ${ }^{\text {a }} 500 \mathrm{ml}$ $20 \%$ lipids [Intralipid ${ }^{\circledR}$ ], $500 \mathrm{ml}$ dextrose $50 \%,{ }^{\text {c }} 2 \mathrm{ml}$ vitamin B complex). Eighteen days after admission, a bilateral tympanic bulla osteotomy was performed. The calf was 
positioned in dorsal recumbency with the neck extended and the head secured to the operating table. The identical procedure was followed for both bullae. The tympanic bulla is located dorsal and medial to the caudal aspect of the vertical ramus of the mandible and the paracondylar process of the occipital bone. A paramedian skin incision was made, centered between these 2 structures. The platysma muscle was incised longitudinally, deep dissection was continued on the medial aspect of the mandibular lymph node, and the digastricus muscle was bluntly separated from the hyoglossal and styloglossal muscles. A periosteal elevator was used to remove soft tissue from the rounded ventral aspect of the bulla. Care was taken to avoid the hypoglossal nerve located on the lateral aspect of the hyoglossal muscle, the external carotid artery dorsal and lateral to the tympanic bulla, and the glossopharyngeal nerve medial to the bulla. After removing the entire ventral aspect of the tympanic bulla with the use of rongeurs, the exudate present in the tympanic cavity was sampled for culture and removed with a curette, warm saline, and suction. Care was taken to avoid damaging the dorsal aspect of the bulla. A mushroom-type drain was placed into the tympanic cavity and exited through the incision. The drain was secured, and the digastricus, hyoglossal, and styloglossal muscles and skin were apposed in a routine manner. Antibiotics and nonsteroidal anti-inflammatory drugs were continued, and the tympanic bullae were lavaged daily with warm saline for 3 days. After confirming Mycoplasma growth from the bullae, oxytetracycline therapy was reinstituted for 6 consecutive days. The calf's neurologic deficits improved dramatically after surgery; she was no longer febrile and was discharged on florfenicol for a total duration of 10 days. At 1 year of age, she was doing well, had normal growth, and did not show any clinical signs.

These cases illustrate clinical signs highly indicative of otitis media/interna and some unexpected aspects. A facial nerve deficit is commonly present because of the proximity of CN VII to the tympanic cavity, ${ }^{1}$ although clinical recognition can be difficult. Ruminants are able to cover the whole cornea with the 3rd eyelid, which seems to prevent ulcerative exposure keratitis. The facial nerve contains parasympathetic fibers that stimulate tear production; however, these leave the facial nerve before the nerve courses through the tympanic cavity, ${ }^{2}$ which could be the reason why decreased tear production is rarely seen in calves and, in fact, epiphora is commonly reported. . $^{3,4}$ Varying degrees of peripheral vestibulocochlear dysfunction are present because of the involvement of the vestibulocochlear receptors and nerve. ${ }^{5}$

Petrous temporal bone osteomyelitis can result in bacterial meningitis and abscess formation in the meninges or brainstem, clinically evident as obtundation. ${ }^{6}$ In 2 of these calves, there was a marked difference between the lumbosacral and the atlanto-occipital CSF cell count, which might indicate that meningeal inflammation was fairly localized, that there was dilution of cells depending on the collection site, or that there was progression of meningitis. This suggests that, for the most accurate assessment, CSF should be collected closest to the suspected site of inflammation when possible.

Spontaneous regurgitation and dysphagia, as seen in

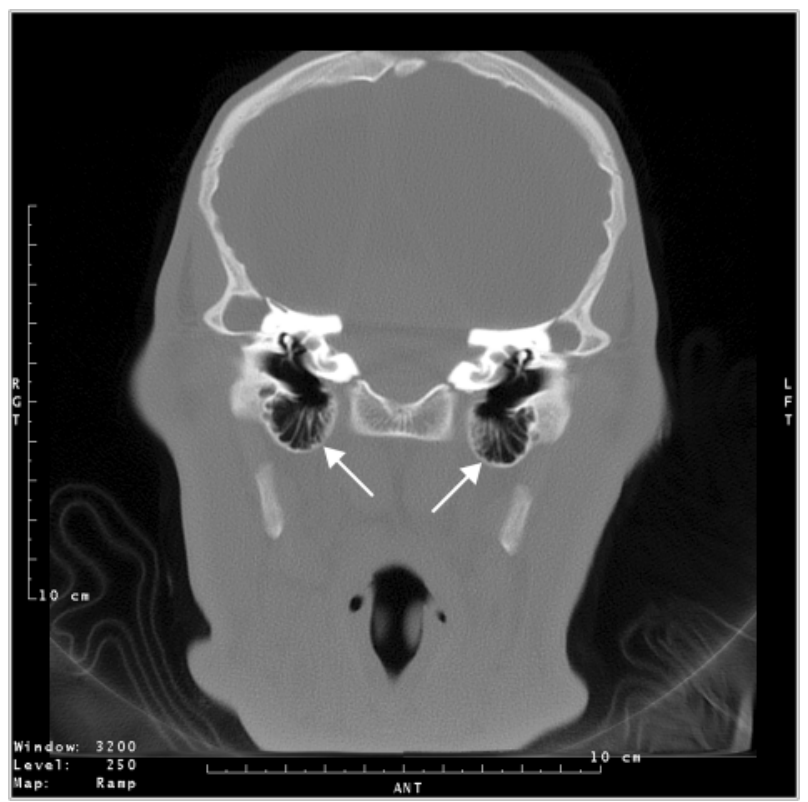

Fig 3. Computed tomographic scan image, bone window, normal calf of 1 month of age. The thin-walled, air-filled bullae with the characteristic bone partitions can be seen (arrows).

these calves, have not been reported previously. Facial nerve paralysis can cause mild ptyalism, but not spontaneous regurgitation and dysphagia. Endoscopic examination in these calves indicated a general loss of pharyngeal tone, dorsal displacement of the soft palate, and esophageal hypomotility and hypotonicity, leading to passive regurgitation of forestomach content. This suggests a lesion involving the glossopharyngeal nerve (CN IX) with or without the vagus nerve (CN X). These nerves could be affected by the inflammation associated with meningitis because both $\mathrm{CN}$ IX and $\mathrm{CN} \mathrm{X}$ travel through the subarachnoid space and leave the cranial vault through the jugular foramen. ${ }^{7}$ The jugular foramen is in close proximity to the internal acoustic meatus, which is the likely focus of meningeal inflammation. Another hypothesis is that CN IX and $\mathrm{X}$ are affected as they pass over the medial, respectively caudal, outer surface of the tympanic bullae. ${ }^{7}$ This would require that the inflammation extends through the wall of the bullae and seems therefore less likely. Pain elicited by jaw movement was thought to be the cause of the difficult prehension and mastication because the inflamed bullae are in close proximity to the temporomandibular joint and there was no evidence for a $\mathrm{CN} \mathrm{V}$ deficit.

CT imaging for otitis media/interna in calves has not been reported but is regarded as the modality of choice in small animals to provide detailed information of the bony structures of the middle and inner ear. ${ }^{8}$ Although oblique radiographs have been reported to be helpful, ${ }^{4} \mathrm{CT}$ evaluation can be expected to be more sensitive than conventional radiography, especially because the bony partitions in the tympanic bulla of the normal calf can decrease the radiographic difference between normal air-filled (Fig 3) and fluid-filled bullae ${ }^{9}$ (Figs 1, 2). The CT findings corresponded well to those seen in small animals ${ }^{8}$ and to the postmortem findings of other reports, in which fluid or exudate accu- 
mulation was found in enlarged tympanic bullae along with mucosal thickening as a result of proliferation of a fibrous inflammatory tissue, osteolysis of the septa, and osteomyelitis of the petrous temporal bone..$^{3,6,9}$ In chronic cases, lesions are extensive and necrosis or sequestration is common. The thickness of the bulla wall should be interpreted with caution because it can appear artifactually thicker in a fluid-filled, than it does in an air-filled, bulla. ${ }^{8}$ Contrast enhancement is expected because of the degree of bone lysis.

Otitis media/interna in calves has a guarded prognosis, ${ }^{3}$ with an estimated mortality rate of $50 \%$. The disease is often detected in late stages when infection is established and can involve the surrounding bone. Long-term intravenous administration of antimicrobials aimed at Mycoplasma spp. and other respiratory pathogens might be required. ${ }^{3,10}$ The poor response to medical therapy of calf 3 could have been due to poor antibiotic penetration or antimicrobial resistance because many strains of $M$ bovis are resistant to oxytetracycline. ${ }^{11}$ Weight loss, as seen in these calves, has been reported in the majority of cases with fatal outcome, 3,9 suggesting that it might have been a significant factor in their demise.

The authors are unaware of any published reports examining the development carrier status after successful treatment of Mycoplasma infections of the respiratory tract, the middle ear, or either, and the risk this poses for introduction of Mycoplasma mastitis in the herd. Control measures for potential concurrent herd problems with Mycoplasma mastitis should be recommended. ${ }^{12}$

\section{Footnotes}

${ }^{a}$ Travasol ${ }^{\circledR}$, Baxter Healthcare Corporation, Clintec Nutrition Division, Deerfield, IL
${ }^{\mathrm{b}}$ Intralipid ${ }^{\circledR}$, Baxter Healthcare Corporation, Clintec Nutrition Division, Deerfield, IL

${ }^{\mathrm{c}}$ Dextrose $50 \%{ }^{\circledR}$, Abbott Laboratories, North Chicago, IL

\section{References}

1. de Lahunta A. Cranial nerve-lower motor neuron: General somatic efferent, special visceral efferent system. In: de Lahunta A, ed. Veterinary neuroanatomy and clinical neurology, 2nd ed. Philadelphia, PA: WB Saunders; 1983:95-114.

2. de Lahunta A. Lower motor neuron-general visceral efferent system. In: de Lahunta A, ed. Veterinary neuroanatomy and clinical neurology, 2nd ed. Philadelphia, PA: WB Saunders; 1983:128.

3. Walz PH, Mullaney TP, Render JA, et al. Otitis in preweaned Holstein dairy calves in Michigan due to Mycoplasma bovis. J Vet Diagn Invest 1997;9:250-254.

4. Francoz D, Fecteau G, Desrochers A, Fortin M. Otitis media in dairy calves: 15 cases from 1987-2002. Proceedings of the 21st Annual Forum ACVIM, Charlotte, NC, June 2003.

5. de Lahunta A. Vestibular system-special proprioception. In: de Lahunta A, ed. Veterinary neuroanatomy and clinical neurology, 2nd ed. Philadelphia, PA: WB Saunders; 1983:238-254.

6. Vestweber JG. Otitis media/interna in cattle. Comp Cont Ed Pract Vet 1999;21:S34-S38.

7. Godinho HP, Getty R. Ruminant nervous system. Peripheral nervous system. Cranial nerves. In: Getty R, ed. Sisson and Grossman's The Anatomy of the Domestic Animals, 5th ed. Philadelphia, PA: WB Saunders; 1975:1081-1093.

8. Garosi LS, Dennis R, Schwarz T. Review of diagnostic imaging of ear diseases in the dog and cat. Vet Rad Ultrasound 2003;44:137146.

9. Jensen R, Maki LR, Lauerman LH, et al. Cause and pathogenesis of middle ear infection in young cattle. J Am Vet Med Assoc 1983; 182:967-972.

10. Nicholas RAJ, Ayling RD. Mycoplasma bovis: Disease, diagnosis, and control. Review. Res Vet Sci 2003;74:105-112.

11. Thomas A, Nicolas C, Dizier I, et al. Antibiotic susceptibilities of recent isolates of Mycoplasma bovis in Belgium. Vet Rec 2003;153: $428-431$.

12. Gonzàlez RN, Wilson DJ. Mycoplasmal mastitis in dairy herds. Vet Clin North Am Food Anim Pract 2003;19:199-221. 